Signal \& Image Processing : An International Journal (SIPIJ) Vol.5, No.3, June 2014

\title{
BEAMFORMING WITH PER-ANTENNA POWER CONSTRAINT AND TRANSMIT ANTENNA SELECTION USING CONVEX OPTIMIZATION TECHNIQUE
}

\author{
Suban and Ann Caroline Jenifer \\ Department of Electronics and Communication Engineering, Velammal College of \\ Engineering and Technology, Madurai.
}

\begin{abstract}
In this paper, transmit beamforming and antenna selection techniques are presented for the Cooperative Distributed Antenna System. Beamforming technique with minimum total weighted transmit power satisfying threshold SINR and Per-Antenna Power constraints is formulated as a convex optimization problem for the efficient performance of Distributed Antenna System (DAS). Antenna Selection technique is implemented in this paper to select the optimum Remote Antenna Units from all the available ones. This achieves the best compromise between capacity and system complexity. Dual polarized and Triple Polarized systems are considered. Simulation results prove that by integrating Beamforming with DAS enhances its performance. Also by using convex optimization in Antenna Selection enhances the performance of multi polarized systems.
\end{abstract}

\section{KEYWORDS}

Distributed Antenna System, Beamforming, Convex Optimization, Antenna Selection, Dual polarized, Triple Polarized.

\section{INTRODUCTION}

Wireless communication systems have been developing and evolving in a furious pace in these recent years. The number of mobile subscribers is growing tremendously in the past decades. The early wireless systems consisted of a base station with a high-power transmitter which serves a large geographic area. Each base station could serve only a small number of users and was costly as well. Today, due to the advancement in technology, the cellular system consists of a cluster of base stations with low-power radio transmitters. Now the total number of users served is increased because of channel reuse and larger frequency bandwidth.

Next generation broadband wireless access systems are evolving towards distributed architectures as a promising solution to meet the ever increasing demand for the wireless connectivity. With limited transmit power and bandwidth; one way to achieve the high data rate is to reduce the radio transmission distance between the transmitter and the receiver which is made possible by using the distributed architectures [1]. It is well established that multiple-input multiple-output DOI : $10.5121 /$ sipij.2014.5306 
Signal \& Image Processing : An International Journal (SIPIJ) Vol.5, No.3, June 2014

(MIMO) technology provides high data rates and link reliability without additional bandwidth or power [2]. Efficient allocation of the transmit power over the coverage area is possible by introducing the MIMO technologies into new radio architectures especially for future mobile communication systems [3]. Distributed Antenna System (DAS) is an evolving architecture which serves the need of the future wireless communication systems.

Beamforming or spatial filtering is a powerful signal processing technique used in antenna arrays or sensor arrays for directional signal transmission or reception. Beamforming helps in main lobe enhancement, side lobe reduction and removal of interference caused by unwanted transmitters.

There are also a set of limitations that result from practical system design such as a Per-Antenna power constraint that keeps the amplifier at each transmit antenna in its linear range [4]. In designing the communication system, these types of constraints should all be taken into account. Instead of Sum power constraint, in this paper we consider the more realistic Per-Antenna power constraint on each of the transmitters at the Remote Antenna Units (RAUs), since in practice each antenna is limited individually by its equipped power amplifier.

In paper [5], the transmitter optimization problem for multiuser downlink channels with multiple transmit antennas at the base-station is considered. Assuming perfect channel knowledge at the transmitter, this paper investigates two different transmission schemes under the Per-Antenna power constraint: a minimum-power beamforming design for downlink channels with a single antenna at each remote user and a capacity-achieving transmitter design for downlink channels with multiple antennas at each remote user. It is shown that in both cases, the Per-Antenna downlink transmitter optimization problem may be transformed into a dual uplink problem with an uncertain noise. This new interpretation of duality gives rise to efficient numerical optimization techniques for solving the downlink Per-Antenna transmitter optimization problem.

The benefit of coordinating base-stations across multiple cells in a multi-antenna beamforming system, where multiple base-stations may jointly optimize their respective beamformers to improve the overall system performance is considered in [6]. This paper focuses on the design criteria of minimizing either the total weighted transmitted power or the maximum per-antenna power across the base-stations subject to Signal-to-Interference-and-Noise-ratio (SINR) constraints at the remote users. The main contribution of this paper is an efficient algorithm, which is capable of finding the globally optimal downlink beamforming vector across all basestations.

\section{COOPERATIVE Distributed ANTENNA SYSTEMS}

The Cooperative Distributed Antenna System (DAS) is a promising system for future wireless communications. Unlike the conventional collocated antenna systems, where antennas are centrally collocated, the Remote Antenna Units (RAUs) of DAS are geographically distributed in the cell. RAUs are simple antenna units which carry out transmission and reception for its Base Station (BS) or Central Unit (CU).RAUs are connected via optical fibers to the central unit (CU) or the BS [1]. Thus it minimizes the communication overheads of system coordination. Complicated signal processing algorithms are performed at the BS. DAS cellular model is shown in Fig.1. 
Signal \& Image Processing : An International Journal (SIPIJ) Vol.5, No.3, June 2014

Since all the RAUs are connected to the BS, spatial diversity can be achieved, thus improving the system performance. Also, having some antennas located closer to the mobile station decreases the average distance of propagation to or from the nearest antenna, thus reducing the required uplink and downlink transmitted power for a fixed channel quality and creating more uniform coverage inside the cell [7]. DAS is often used in scenarios where alternate technologies are infeasible due to terrain or zoning challenges. The idea works because less power is wasted in overcoming penetration and shadowing losses, and because a line-of-sight channel is present more frequently, leading to reduced fade depths and reduced delay spread. In the cooperative DAS the transmitted signals of a few RAUs, instantaneously linked to a reference MT, are considered useful signals rather than interference.

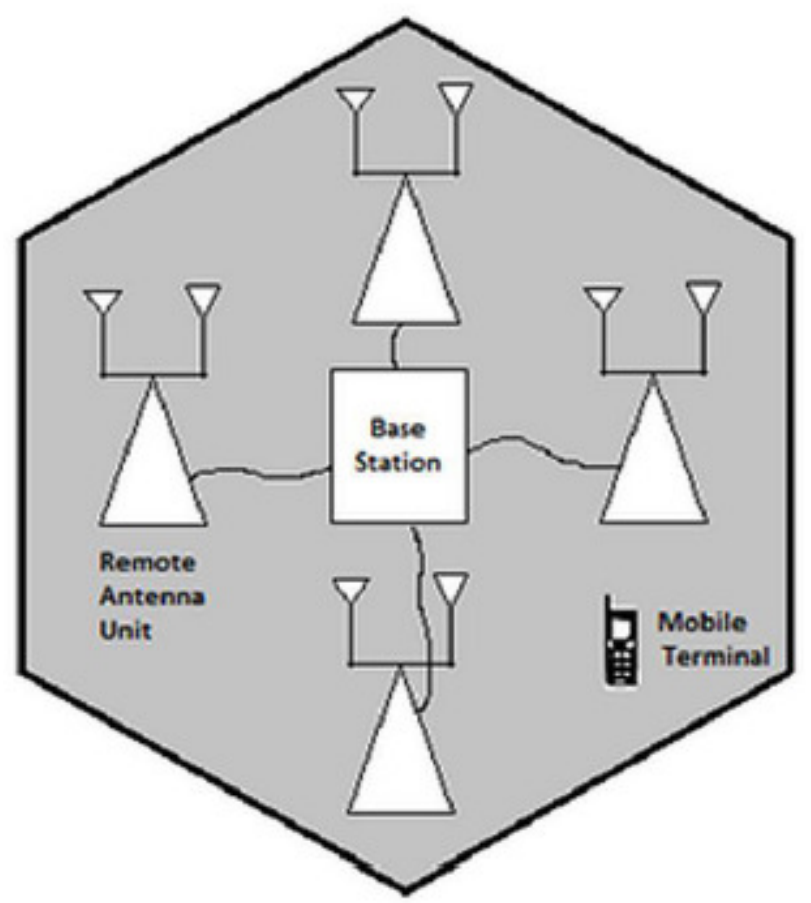

Fig.1. DAS cellular model

DAS was originally proposed to improve the indoor performance of wireless communication systems. In order to exploit the advantages of both the collocated MIMO and the DAS, a cooperative DAS based on a radio over fiber (RoF) technique was introduced in China's beyond $3 \mathrm{G}$ FuTURE project $[8,9]$ and tested via field experiments. Research on distributed MIMO has been carried out in [10-12] by exploiting both microscopic and macroscopic spatial diversity. In [3] an analytical capacity study was presented for DASs.

A set of RAUs within reachable distances of a reference MT can cooperatively communicate with this MT to form a MIMO system. Note that in cooperative DAS the distributed MIMO system differs from the traditional centralized MIMO since each of the RAUs experiences independent macroscopic fading from each other [11]. Therefore, the distributed MIMO formed by several RAUs at different geometric locations can make use of the statistically independent properties of the channels more efficiently, and larger channel capacity would be expected than in the traditional collocated MIMO. The theoretic and Monte Carlo simulation results presented in [3] shows the benefits of the distributed MIMO. 
Signal \& Image Processing : An International Journal (SIPIJ) Vol.5, No.3, June 2014

\section{BEAMforming AND PER-Antenna POWER Constraint}

Beamforming is a signal processing technique used in antenna arrays for directional transmission and reception. It steers all the energy in one direction by directing a transmitting element in an antenna array to reach a desired receiver in a given direction. Using Beamforming technique, weight vectors are assigned for each channel. The following assumptions are made 1) Perfect Channel State Information at the transmitter (CSIT) 2) The channel is assumed to be a frequency flat quasi static channel 3) The number of users are greater than the number of transmit antennas at the RAU.

Depending upon the CSIT, Beamforming technique allocates weight vectors for each channel. Now, the transmit antenna element in the RAU whose channel is assigned a high value of weight vector is chosen from among the available set of antenna elements and transmission takes place between the selected antenna terminal in the RAU and the mobile terminal through this channel.

Also in practical multi-antenna implementations, each transmit antenna is usually equipped with its own power amplifier. Thus, an individual power constraint on each antenna individually is more realistic than a sum power constraint across all the antennas. There are certain benefits in considering the Per-Antenna power constraint. They are as follows

- It helps in joint transmission and reception.

- Since the power is equally divided among the transmit antenna elements, all the antennas will be active at a particular time.

Hence in this paper we consider the Per-Antenna power constraint.

\section{SYSTEM MODEL}

Consider a DAS scenario where a Base Station (BS) is located at the center of the macro cell and the multiple antennas of the BS, the Remote Antenna Units (RAUs) are distributed throughout the cell. The RAUs are connected to the BS by an optical fiber or cable. Each RAU consists of one or more antenna terminals. Mobile Terminals (MTs) can be located anywhere in the cell. The system model is shown in Fig.2.

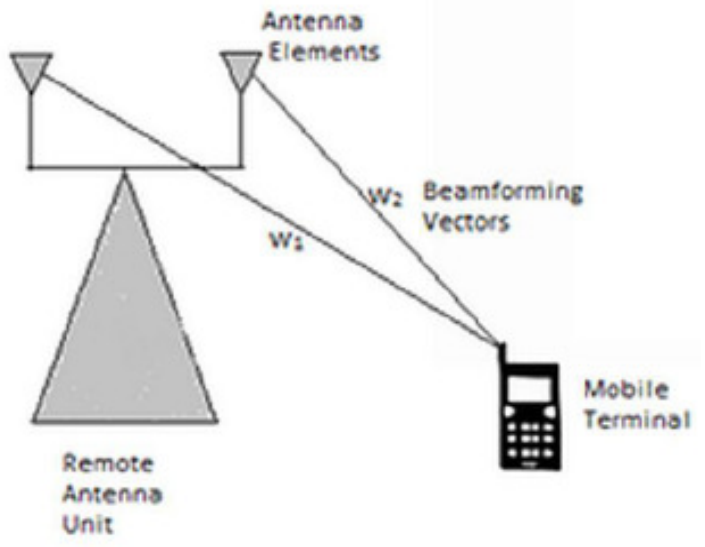

Fig.2. System Model (showing a RAU with 2 antenna elements and a MT with 1 antenna) 
The general system model for a RAU with $\mathrm{N}$ antenna elements and $\mathrm{K}$ receivers, each with one antenna can be given as,

$$
y_{k}=\mathbf{w}_{\mathbf{k}}^{\mathrm{H}} \mathbf{h}_{\mathbf{k}}+n_{k}, \quad k=1,2, \ldots \ldots . K
$$

where, $\mathrm{y}_{\mathrm{k}}$ represents the received signal $(1 \mathrm{x} 1), \mathbf{w}_{\mathrm{k}}^{\mathrm{H}}$ is a $(1 \mathrm{xN})$ beamforming vector, $\mathrm{h}_{\mathrm{k}}$ is a $(\mathrm{Nx} 1)$ channel response vector and $\mathrm{n}_{\mathrm{k}}$ represents the noise $(1 \mathrm{x} 1)$.

The channel capacity equation of the distributed antenna system is given as [15].

$\mathrm{C}(\mathrm{G})=\log _{2} \operatorname{det}(\mathrm{IM}+\rho \mathrm{M} \mathrm{D})$

Where $\mathrm{G}$ is the downlink propagation channel, $\rho$ is the SINR, D is the Large Scale Fading matrix and $\mathrm{M}$ is the number of RAUs.

\section{BEAMFORMing in DAS AS A CONVEX OPTIMIZATION PROBlem}

Let $\mathrm{w}_{\mathrm{k}}$ denote the weight vectors $(\mathrm{k}=1,2 \ldots \mathrm{K})$. The total weighted power at the transmitter is given as $\sum_{k=1}^{K}\left\|\mathrm{w}_{\mathrm{k}}\right\|^{2}$. Increasing the transmit power affects the linearity of devices (e.g. amplifier) present in the RAU. This is an undesirable effect. Hence the aim is to reduce the total weighted transmit power but at the same time ensure that the Signal to Interference-Noise ratio (SINR) target is met at the receiver. We formulate the Beamforming in cooperative DAS as a convex optimization problem to get the solution. Convex optimization technique finds the weight vector that minimizes the total transmit power and also meets the SINR target. The two constraints to be satisfied are the Per-Antenna power constraint and multiuser transmit beamforming under individual SINR constraint. The problem can be expressed as follows

$$
\begin{gathered}
\min _{\left\{\mathbf{w}_{\mathbf{k}} \in C^{5}\right\}_{k=1}^{K}} \sum_{k=1}^{K}\left\|\mathbf{w}_{\mathbf{k}}\right\|^{2} \\
\text { subject to } \frac{\left|\mathbf{w}_{\mathbf{k}} \mathbf{h}_{\mathbf{k}}\right|^{2}}{\sum_{k \neq k}\left|\mathbf{w}_{1}^{\mathrm{H}} \mathbf{h}_{\mathbf{k}}\right|^{2}+\sigma_{k}^{2}} \geq c_{k}, \quad k=1,2, \ldots \ldots, K \\
\mathbf{w}_{\mathbf{k}}=1 / \mathrm{N}
\end{gathered}
$$

Eqn (2) defines the objective function (minimize transmit power), (3) defines the SINR constraint and (4) defines the Per-Antenna power constraint. $\mathrm{N}$ denotes the number of transmit antennas, $\mathrm{c}_{\mathrm{k}}$ denotes the target SINR, $\sigma_{k}^{2}$ denotes the noise power. The problem becomes infeasible when 1) the channel vectors of two or more channels are co-linear or highly correlated 2) SINR target is too high 3) the number of users, $\mathrm{K}$ is much greater than the number of antenna terminals, $\mathrm{N}$ in the RAU. 
Signal \& Image Processing : An International Journal (SIPIJ) Vol.5, No.3, June 2014

\section{Transmit AnTENna SELECTION IN DAS AS A CONVEX OPTIMIZATION PROBLEM}

Antenna Selection is a powerful signal processing technique that can reduce the cost and complexity of RF chains associated with each RAU, but at the same time preserves the diversity and multiplexing gains obtained from the actual system. Also it reduces the signalling burden overhead at the BS. The transmit antenna selection problem in DAS can be formulated as a linear programming problem and can be solved using the Interior Point algorithm. Linear programming is a mathematical optimization technique to solve a linear objective function, subjected to the linear equality and linear inequality constraints. Define $\Delta_{i}(i=1,2 \ldots \mathrm{M})$ as the antenna selection variable for each RAU such that,

$$
\begin{aligned}
\Delta_{i}= & \left\{1, i^{\text {th }} \mathrm{RAU}\right. \text { is selected } \\
& 0, \text { otherwise }
\end{aligned}
$$

Define a M X M diagonal matrix $\Delta$ for RAU selection at the Base Station which has $\Delta_{\mathrm{i}}$ as its diagonal entries. The diagonal matrix $\Delta$ is represented as follows,

$$
\Delta=\left[\begin{array}{llll}
\Delta_{1} & & & \\
& \Delta_{2} & & \\
& & \Delta_{M} & \\
& & & \Delta_{M x M}
\end{array}\right]
$$

The modified channel capacity equation as a function of antenna selection variable can be written as

$$
\mathrm{C}(\Delta)=\log 2 \operatorname{det}(\mathrm{IM}+\rho \mathrm{M} \Delta \mathrm{D})
$$

The set of RAUs that maximizes the capacity of the system must be selected out of all the available RAUs. The antenna selection problem is formulated as a convex optimization problem. The objective function is to maximize the channel capacity. The two constraints defined are: 1) 0 $\leq \Delta \leq 1$, the antenna selection variable is relaxed to a weaker constraint so that it can be solved in polynomial time. 2) the sum of the diagonal elements of $\Delta$ must be equal to $\mathrm{N}$, the total number of RAUs selected. The problem can be expressed as follows,

$$
\begin{gathered}
\text { maximize } \mathrm{C}(\Delta)=\log _{2} \operatorname{det}\left(\mathrm{I}+\mathrm{c}_{\mathrm{k}} \mathrm{M} \Delta \mathrm{D}\right) \\
\text { subject to } 0 \leq \Delta \leq 1 \\
\operatorname{trace}(\Delta)=\sum_{i=1}^{M} \Delta_{i}=N
\end{gathered}
$$

The solution to this problem would be that $\mathrm{N}$ optimal RAUs will be selected from the available set to serve a particular user. 


\section{RESULTS AND DISCUSSION}

For the performance evaluation, the simulation parameters are set as follows: Number of users $K=2$, Number of transmit antennas in the RAU $M_{t}=2$, Number of receive antennas $M_{r}=1$, SINR target $c_{k}=2 \mathrm{~dB}$. Two cases are taken to evaluate the formulated problem. Case 1: Two orthogonal channels are taken. Case 2: Two highly correlated channels are taken. In Case 1, weight vectors are found for link 1 and link 2. By using convex optimization technique, the optimized value of the weight vector is found to be 0.7068 . Optimal value of transmit power is found to be 2.8280 $\mathrm{dB}$. Table 1 tabulates the optimized values obtained for Case 1.

Table I

Optimal Values Obtained for Case 1

\begin{tabular}{|c|c|c|c|}
\hline S.NO & PARAMETERS & \multicolumn{2}{|c|}{ VALUES } \\
\hline 1 & Optimal Value & \multicolumn{2}{|c|}{+2.82805} \\
(cvx_optval) & \multicolumn{2}{|c|}{} \\
\hline 2 & SINR & Link 1 & Link 2 \\
\cline { 3 - 4 } & & 1.9985 & 1.9985 \\
\hline 3 & Weight Vectors & Link 1 & Link 2 \\
\cline { 3 - 4 } & & 0.7068 & 0.7068 \\
\cline { 3 - 4 } & & 0.7068 & -0.7068 \\
\hline 4 & \multirow{2}{*}{$\begin{array}{c}\text { Total transmit } \\
\text { power }\end{array}$} & \multicolumn{2}{|c}{2.8280} \\
& & \multicolumn{2}{|c}{} \\
\hline
\end{tabular}

In Case 2, two channels which are highly correlated are taken. As discussed before, this case becomes infeasible. Hence no weight vectors are assigned. Simulation results for SNR in dB vs. average Bit Error Rate (BER) is shown in Fig.3. Graphs are obtained for four different cases i) $1 \times 1$ MIMO ii) 2x2 MIMO iii) 2x2 MIMO-BF (Beamforming) iv) 4x4 MIMO-BF.

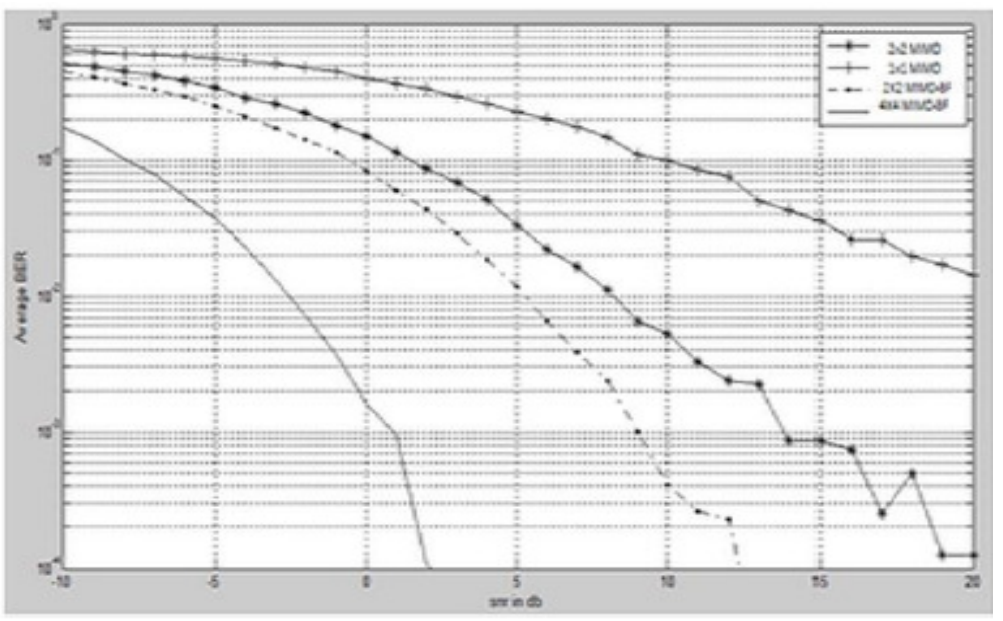

Fig.3.Plot of SNR in dB vs. Average BER 
Signal \& Image Processing : An International Journal (SIPIJ) Vol.5, No.3, June 2014

It is evident from the graph that to attain a minimum average BER of 10-1, SNR needed by the $1 \mathrm{x} 1 \mathrm{MIMO}$ and 2x2 MIMO are $10 \mathrm{~dB}$ and $2 \mathrm{~dB}$ respectively. Thus by increasing the number of transmit and receive antennas, a minimized average BER can be obtained at a reduced SNR. Also including Beamforming technique with MIMO further reduces the SNR (and hence the transmit signal power) needed to achieve a minimized average BER. From Fig.3 we can see that to get a minimum average BER of $10-1$, SNR needed by $2 \times 2$ MIMO-BF and $4 \times 4$ MIMO-BF are $-1 \mathrm{~dB}$ and $-7.5 \mathrm{~dB}$. Table 2 shows the SNR values obtained for different system configurations.

Table 2 Comparison of SNR Values for Different System Configurations

\begin{tabular}{|c|c|c|c|}
\hline \multirow[t]{2}{*}{ S.NO } & \multirow[t]{2}{*}{ SYSTEM } & $\begin{array}{c}\text { MIMO } \\
\text { (Without } \\
\text { Beamforming) } \\
\text { (SNR dB) }\end{array}$ & $\begin{array}{c}\text { MIMO-BF } \\
\text { (With } \\
\text { Beamforming) } \\
\text { (SNR dB) }\end{array}$ \\
\hline & & \multicolumn{2}{|c|}{$\left(\mathrm{BER}=10^{-1}\right)$} \\
\hline 1 & $1 \mathrm{X} 1$ & 10 & - \\
\hline 2 & $2 \mathrm{X} 2$ & 1 & -1 \\
\hline 3 & $4 \mathrm{X} 4$ & - & -7.5 \\
\hline
\end{tabular}

Simulation result for SINR in dB vs. BER for two methods- sum-power constraint and perantenna power constraint is shown in Fig.4.From the plot it is seen that the performance of perantenna power constraint is better than the sum-power constraint. Therefore by implementing the more realistic Per-Antenna power constraint, the amplifiers at the RAU are kept in the linear range. Thus it is clear that by integrating Beamforming techniques with DAS, a reduced total transmit power can be achieved with target SINR constraint. Also, it is clear that Beamforming technique in DAS with per-antenna power constraint achieves a better performance.

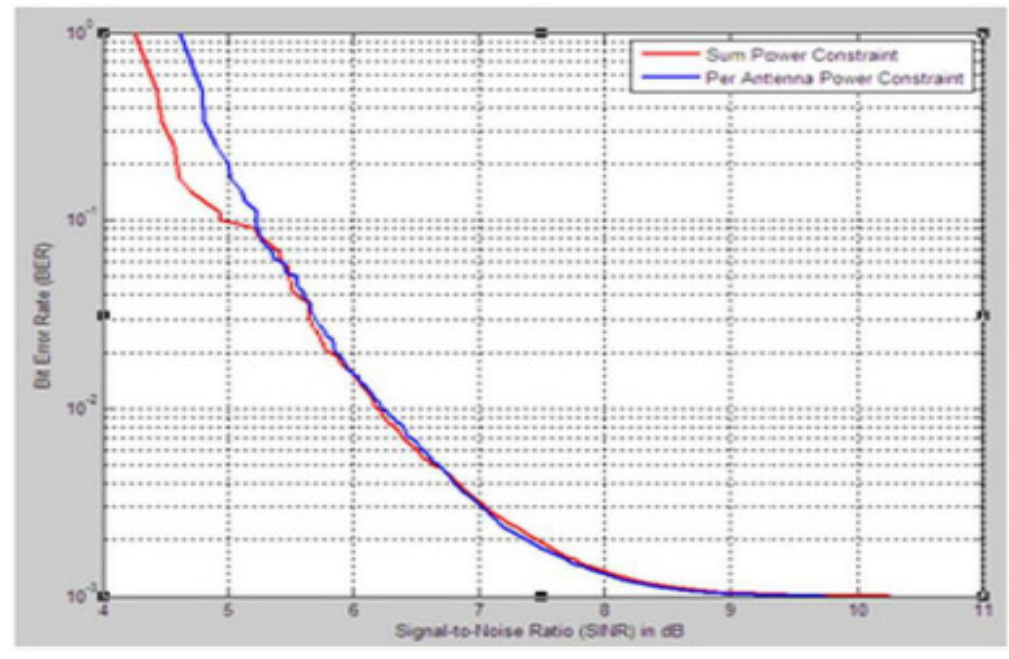

Fig.4. Plot of SINR in dB vs. BER 
Signal \& Image Processing : An International Journal (SIPIJ) Vol.5, No.3, June 2014

Simulation result between Selected RAUs and channel capacity without Antenna Selection is shown in Fig.5. From this figure it is observed that the performance of 2SS-DP and 3SS-SP are similar.

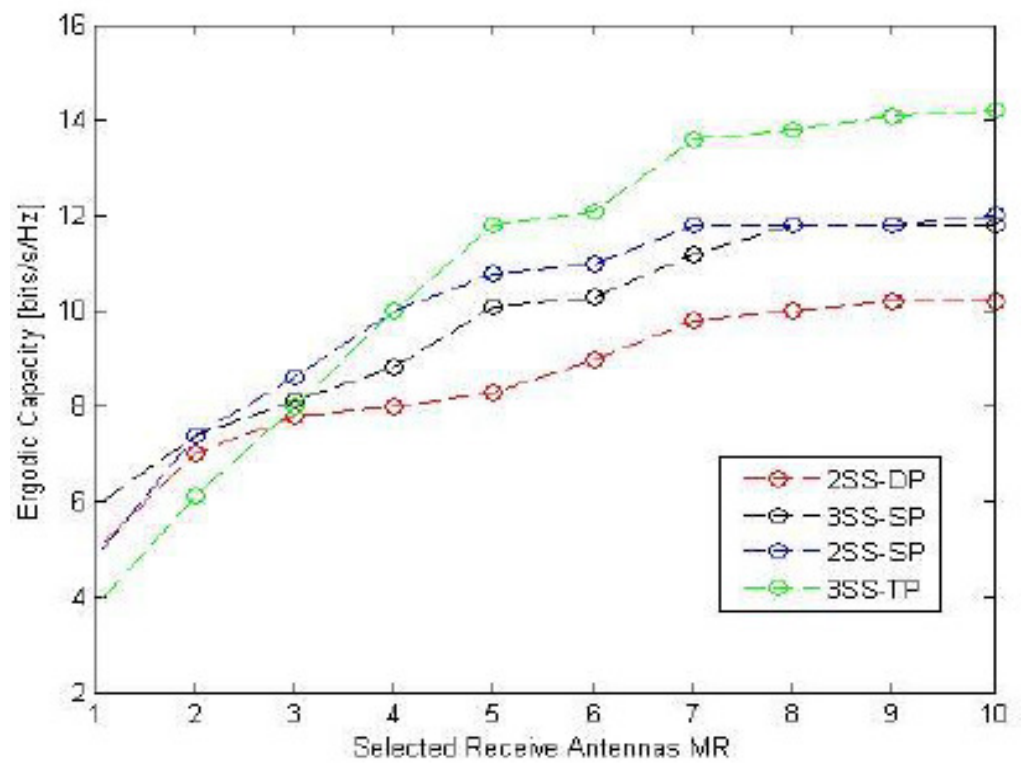

Fig.5. Plot of Selected Antennas vs. Capacity (without Antenna Selection)

Simulation results between Selected Antennas vs. Channel capacity with Antenna Selection is shown in Fig.6. It is evident from the two plots that the performance of Dual Polarized and Triple Polarized systems is enhanced by using Antenna Selection Techniques.

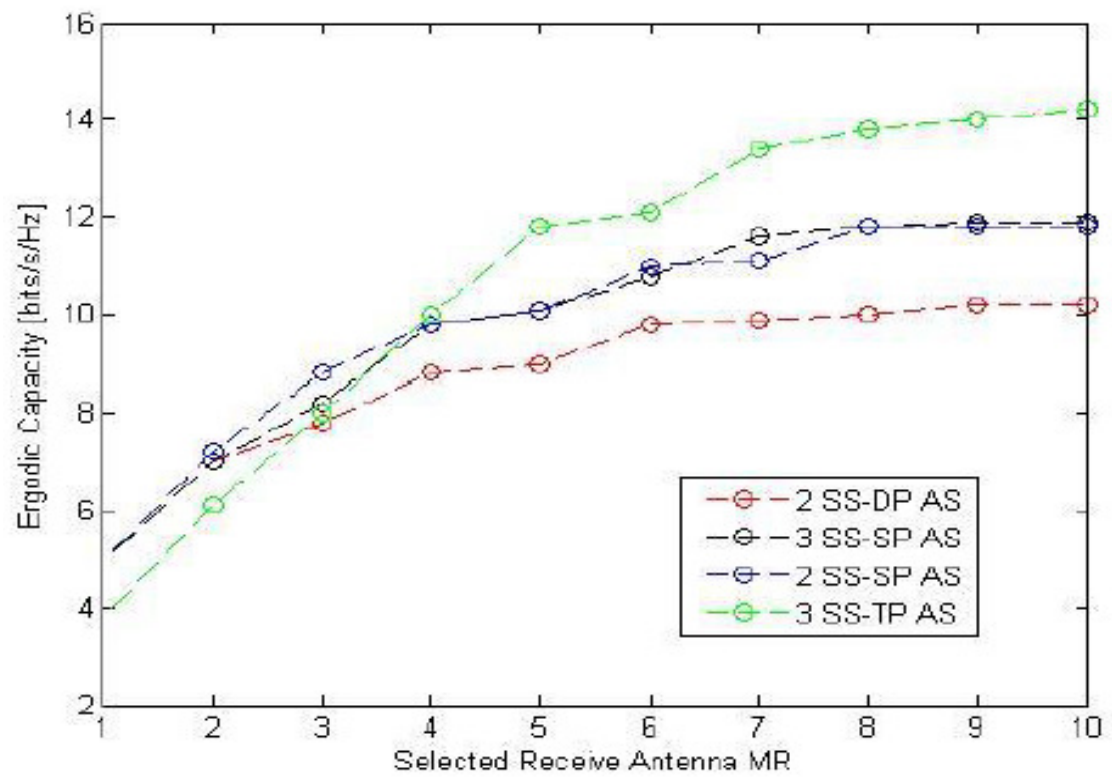

Fig.6. Plot of Selected Antennas vs.Capacity (with Antenna Selection) 
Signal \& Image Processing : An International Journal (SIPIJ) Vol.5, No.3, June 2014

\section{CONCLUSION}

The problem to find the optimal beamforming weights which minimizes the total weighted transmit power, satisfying the SINR constraint and Per-Antenna power constraint, is formulated as a convex optimization problem and is solved using convex optimization tools (e.g. CVX). Two different cases- orthogonal channels and highly correlated channels are discussed. Comparison between Sum power and Per-Antenna power constraint is done. The results and graphs obtained prove that the performance of the system is improved by integrating beamforming technique with Cooperative Distributed Antenna System (DAS). Also convex optimization is used to optimize the performance of DAS for maximizing the ergodic capacity. The optimum RAUs that maximizes the channel capacity is selected by using optimization algorithm. The results are also compared with spatially separated single polarized systems. The performance of multi polarized system is enhanced by using convex optimization. Also the performance of triple polarized system is found to be good under certain channel conditions.

\section{REFERENCES}

[1] H.Zhu, "On Frequency Reuse in Cooperative Distributed Antenna System", IEEE Communications Magazine, vol. 50, No. 4, pp.85-89, April 2012.

[2] S.Mahboob, R.Ruby and V.C.M. Leung, "Transmit Antenna Selection for Downlink Transmission in a Massively Distributed Antenna System using Convex Optimization", in Proceedings of Seventh International Conference on Broadband, Wireless Computing, Communication and Applications, 2012, pp. 228-233.

[3] X.H.You, D.M.Wang, B.Sheng, X.Q.Gao, X.S. Zhao, and M.Chen, "Cooperative Distributed Antenna Systems for Mobile Communications", IEEE Wireless Communications, vol. 17, pp. 33-43, June 2010.

[4] M. Khoshnevisan and J.N. Laneman, "Power Allocation in Multi-Antenna Wireless Systems Subject to Simultaneous Power Constraints", IEEE Transactions on Communications, vol. 60, pp 3855-3864, Dec. 2012.

[5] W.Yu and T.Lan, "Transmitter Optimization for the Multi-Antenna Downlink with Per-Antenna Power Constraints", IEEE Transactions on Signal Processing, vol.55, pp. 2646-2660, June 2006.

[6] H.Dahrouj, W.Yu, "Coordinated Beamforming for the Multicell Multi-Antenna Wireless System", IEEE Transactions on Wireless Communications, vol.9, pp. 1748-1759, May 2010.

[7] R.Heath, S.Peter, Y.Wang and J.Zhang, "A Current Perspective on Distributed Antenna Systems for the Downlink of Cellular Systems", IEEE Communication Magazine, vol. 51, pp. 161-167, April 2013.

[8] X.H.You and X.Q.Gao, "Development of Beyond 3G Techniques and Experiment System: An Introduction to the FuTURE project", in ICT Shaping the World: A Scientific View, Wiley, Nov. 2008.

[9] X.H.Yu, G.Chen, M.Chen and X.Gao, "Toward Beyond 3G: the FuTURE Project in China", IEEE Communications Magazine, vol.43, pp.70-75, Jan.2005.

[10] W.Choi and J.G.Andrews, "Downlink Performance and Capacity of Distributed Antenna Systems in a Multicell Environment", IEEE Transactions on Wireless Communication, vol. 6, pp. 69-73, Jan. 2007.

[11] H. Hu, Y. Zhang, and J. Luo, Distributed Antenna Systems: Open Architecture for Future Wireless Communications, CRC Press, June 2007.

[12] D.M.Wang, X.You, J.Wang and Y.Wang, "Spectral Efficiency of Distributed MIMO Cellular Systems in a Composite Fading Channel," in Proceedings of IEEE International Conference on Communications ICC' 08, 2008, pp.1259-64. 
Signal \& Image Processing : An International Journal (SIPIJ) Vol.5, No.3, June 2014

[13] F. Boccardi and H. Huang, "Optimum power allocation for the MIMO BC zero-forcing precoder with per-antenna power constraints", 2006 40th Annual Conference on Information Sciences and Systems. pp 504, March 2006.

[14] S-R.Lee, S.H.Moon, "Optimal Beamforming Schemes and its Capacity Behavior for Downlink Distributed Antenna Systems", IEEE Transactions on Wireless Communications, vol. 12, no. 6, pp. 2578-2587, June 2013.

[15] F. Rusek, D. Persson, B. K. Lau, E. G. Larsson, O. Edfors, F. Tufvesson,and T. L. Marzetta, "Scaling up MIMO: opportunities and challenges with very large arrays," IEEE Signal processing Magazine, 2012.

\section{AUTHORS}

A. Suban, received B.E in the department of Electronics and Communication Engineering from Anna university, Chennai and M.E in the discipline of Wireless Technology from Thiagarajar College of Engineering, Madurai in 2011. He is currently working as Assistant Professor in the Department of Electronics and Communication Engineering, Velammal College of Engineering and Technology, Madurai-625009, Tamil Nadu, India. His area of interest in Signal processing mainly focused on MIMO techniques with beamforming, OFDM and power control techniques.

R.Ann Caroline Jenifer is currently pursuing her M.E degree in Communication Systems at Velammal College of Engineering, Madurai. She received her B.Tech degree in Electronics and Communication Engineering from Karunya University, Coimbatore in 2012. Her area of interest is Digital Signal Processing.
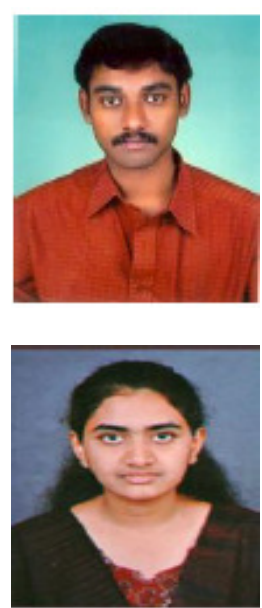